\title{
Die neue Schweizer Gesetzgebung zu bewegungseinschränkenden Maßnahmen auf dem medizinethischen Prüfstand
}

\author{
Wiebke Bretschneider
}

Eingegangen: 3. Mai 2013 / Angenommen: 15. Mai 2014 / Online publiziert: 30. Juli 2014

(C) Springer-Verlag Berlin Heidelberg 2014

Zusammenfassung Bewegungseinschränkende Maßnahmen werden in verschiedenen medizinischen Institutionen angewendet, um das Verhalten von Patienten durch physische bzw. mechanische oder chemische Eingriffe zu beeinflussen. Solche Maßnahmen stellen eine Art Freiheitsentziehung dar und bedürfen daher immer einer Rechtfertigung. Die Frage nach der Indikation für bewegungseinschränkende Maßnahmen ist abzuklären, da ein standardmäßiger Gebrauch nicht Ziel einer Behandlung sein sollte. Gesetze können als Richtschnur für behandelnde Ärzte und Pflegepersonal dienen. In der Schweiz ist am 1. Januar 2013 erstmals eine gesetzliche Regelung zu bewegungseinschränkenden Maßnahmen eingeführt worden. Hauptziel dieser neuen Artikel im Schweizerischen Zivilgesetzbuch (Artikel 383 ff. ZGB) ist die Stärkung von Autonomie und persönlicher Freiheit von Patienten. Es ist wichtig, Vorschriften zu hinterfragen bzw. deren Auswirkungen für die betroffenen Personen zu analysieren und einen kritischen Dialog zu führen. Die vorliegende Publikation betrachtet bewegungseinschränkende Maßnahmen aus zwei verschiedenen Perspektiven: einerseits anhand einer medizinethischen Debatte, andererseits anhand einer Analyse der gesetzlichen Regelung in der Schweiz. Die neuen Artikel umfassen eine detaillierte Regelung zur „Einschränkung der Bewegungsfreiheit“. Dennoch schließen sie nicht alle bestehenden Regelungslücken. Die neue Schweizer Gesetzgebung kann daher nur als ein erster Schritt in die richtige Richtung betrachtet werden. Ausführungen zur praktischen Anwendung sind dringend nötig und sollten so schnell wie möglich ausgearbeitet werden.

Schlüsselwörter Bewegungseinschränkende Maßnahmen · Fixierung · Patienten · Schweizerische Rechtslage $\cdot$ Autonomie $\cdot$ Pflege

\footnotetext{
W. Bretschneider $(\bowtie)$

Institut für Bio- und Medizinethik, Universität Basel,

Bernoullistr. 28,

4056 Basel, Schweiz

E-Mail: wiebke.bretschneider@unibas.ch
} 


\title{
The new Swiss regulation on restraint measures on medico-ethical trial
}

\begin{abstract}
Definition of the problem Restraint measures are still used in different medical institutions to change or influence patients' behaviour. Arguments Mobility restrictions can constitute a deprivation of liberty and justification is always needed. Also, indications for these measures have to be clarified as the aim of treatment in patients' health care should not be their standard application. Regarding this issue, legal regulations can be guidance for physicians in charge, the nursing staff and a supportive tool in times of uncertainty. For the first time, on 1 January 2013, a law addressing restraint measures came into force in Switzerland. The main aim of this new regulation (article 383 et seqq. ZGB) is to strengthen autonomy and personal freedom of patients. Nevertheless, it is highly important to engage in a dialogue and to question rules like this. Not only is awareness important, but the consequences for all involved persons need to be examined. The objectives of this paper are to illuminate restraining measures from two different perspectives. This will be done by a medico-ethical debate as well as by analysing the new legal regulation in Switzerland. Conclusion Even though the introduced articles provide a detailed regulation about "Restrictions of freedom of movement", not all existing regulatory gaps are filled. The new Swiss legislation can only be called a first step in the right direction. More information on the practical application is urgently needed and should be developed as soon as possible.
\end{abstract}

Keywords Restraint measures $\cdot$ Restraint $\cdot$ Patients $\cdot$ Swiss law $\cdot$ Autonomy $\cdot$ Care

\section{Einleitung}

Bewegungseinschränkende Maßnahmen bei Patienten haben eine lange „Tradition“. Schon im Altertum wurden magisch-religiöse Heilmethoden eingesetzt, um geistige Erkrankungen mit Hilfe von Drogen und Beschwörungen zu heilen. Historiker vermuten, dass Patienten um 1000 v. Chr. bereits mit Opium betäubt wurden [1]. Im Mittelalter kam es dann zu einem vermehrten Gebrauch dieser Praktik. Patienten wurden nicht mehr nur mit pflanzlichen Mitteln oder Medikamenten ruhig gestellt, sondern es wurde auf mechanische Hilfsmittel zurückgegriffen. Patienten wurden bspw. mit Zwangsstühlen, -jacken, Körpergurten oder Halsmanschetten am Bewegen gehindert. Im 17. Jh. verringerte sich der Gebrauch von körpernahen Fixierungen und betroffene Personen wurden vor allem in Gefängnissen und Armenhäusern untergebracht. Erst im 19. Jh. kam es zu einer Minimierung des Gebrauches von bewegungseinschränkenden Maßnahmen jeglicher Art [45]. Trotz dieser Wahrnehmungsänderung sind ,humane Fixierungen“ bzw. Maßnahmen, welche die physische Bewegungsfreiheit von Patienten einschränken, seit vielen Jahren eine verbreitete Praxis [27]. Auch in der Schweiz war der demütigende Umgang mit Pflegebedürftigen zumindest bis ins 20. Jh. eine alltägliche Situation [54]. In der wissenschaftlichen Literatur zeichnet sich jedoch in den letzten Jahren eine zunehmend kritische Haltung gegenüber der Vornahme von Fixierungsmaßnahmen ab [6]. Flaherty geht sogar so weit zu sagen, dass eine medizinische Versorgung ohne Fixierungen der normative Standard sein sollte [16].

In der heutigen Zeit sind vor allem Menschen mit demenziellen Erkrankungen von bewegungseinschränkenden Maßnahmen betroffen [22]. Die ausgesprochene Vulnerabilität bestimmter Patientengruppen macht es besonders wichtig, Umstände und Rechtfertigungs- 
gründe für Bewegungseinschränkungen zu untersuchen. Wie die Inhalte verschiedener Artikel $[19,40]$ sowie die Notwendigkeit der In-Kraft-Setzung neuer gesetzlicher Regelungen zeigen, sind - trotz zunehmend kritischer Einwände - bewegungseinschränkende Maßnahmen nicht gänzlich aus Pflegeheimen, Krankenhäusern und/oder Privathaushalten verbannt. In der Schweiz ist zum 1. Januar 2013 im Rahmen der Revision des Schweizerischen Zivilgesetzbuches (ZGB) erstmals eine eidgenössische Regelung zu bewegungseinschränkenden Maßnahmen eingeführt worden. Bis zu diesem Zeitpunkt waren lediglich medizinische Richtlinien oder allenfalls interne Regularien vorhanden, welche die Vornahme von bewegungseinschränkenden Maßnahmen bei Patienten geregelt haben.

\section{Definition von bewegungseinschränkenden Maßnahmen}

Um die Vornahme von bewegungseinschränkenden Maßnahmen beurteilen zu können, muss zuerst die Bedeutung des Begriffs geklärt werden. Dazu kann als Anhaltspunkt eine im Jahr 2005 veröffentlichte Richtlinie der Schweizerischen Akademie der Medizinischen Wissenschaften herangezogen werden. In dieser heißt es: „Von Freiheitsbeschränkung spricht man, wenn ausschließlich die Bewegungsfreiheit eingegrenzt wird (z. B. die Unterbringung in einer geschlossenen Abteilung). Schwerwiegende Freiheitsbeschränkungen sind die Fixierung (z. B. mit Gurten) oder die Isolation (z. B. in einem Isolierzimmer)“ [46].

Bei dem genannten Begriff der Fixierung handelt es sich um mechanische Bewegungseinschränkungen, zu denen körpernahe Freiheitsbeschränkungen mit Westen für den Oberkörper sowie Riemen oder Gurte für Becken, Hand- oder Fußgelenke gehören [4, 21]. Bettgitter sind keine körpernahen Fixierungen, zählen aber gleichfalls zu mechanischen Bewegungseinschränkungen. Jedoch werden sie in vielen Studien und wissenschaftlichen Artikeln zu Fixierungsmaßnahmen nicht einbezogen [35, 38]. Gurte, um einen Patienten am Bett zu fixieren, werden vom Pflegepersonal als restriktivste Maßnahme empfunden. Einseitige Bettgitter und Sensormatten dagegen als am wenigsten einschneidende Maßnahmen, während beidseitige Bettgitter als moderat eingestuft werden [23]. Die mechanische Bewegungseinschränkung ist die wohl am meisten verbreitete Form der Fixierung im medizinischen und pflegerischen Alltag. Daneben gibt es noch eine weitere Form: die chemische Bewegungseinschränkung. Die Vergabe von Sedativa, Neuroleptika und angstlösenden Medikamenten kann auch eine bewegungseinschränkende Maßnahme sein, wenn sie bewusst zur Verhaltenskontrolle eingesetzt wird [28]. Aufgrund der unterschiedlichen Wirkmodi [13] und der Schwierigkeit zu unterscheiden, ob es sich um eine indizierte Medikation handelt oder die Medikation allein dazu dient, Bewegungen des Patienten zu verhindern, wird diese Art der Fixierung oft aus Studien ausgeschlossen [36]. Die Autonomie, die Freiheit und das Recht des Patienten, Risiken einzugehen, werden hingegen durch beide Fixierungsarten eingeschränkt [34]. Außerdem haben sie die Verhaltenskontrolle von Patienten gemein. Die mechanische und chemische Form der Fixierung unterscheidet jedoch der Weg dorthin. Während bei mechanischen Bewegungseinschränkungen die physische Bewegungseinschränkung im Vordergrund steht, ohne direkt Einfluss auf die mentalen Fähigkeiten des Patienten zu nehmen, hat die chemische Fixierung eine ganzheitliche Wirkung auf den Körper und schränkt den Patienten physisch und psychisch ein. Laut einer Studie werden chemische Fixierungen vor allem bei Patienten mit aggressivem Verhalten eingesetzt, wohingegen mechanische vorrangig zum eigenen Schutz des Patienten, 
bspw. vor Stürzen, verwendet werden [47]. Häufig wird allein die mechanische Fixierung als eine echte Fixierung wahrgenommen [20]. Das fehlende Bewusstsein darüber, welche Maßnahmen in die Kategorie der Fixierung fallen, verdeutlicht die Notwendigkeit der Sensibilisierung für bewegungseinschränkende Maßnahmen. Nur mithilfe klarer Definitionen und der Klärung der Begrifflichkeiten ist dies möglich [29].

Neben dementiellen Erkrankungen zählen Aufgeregtheit, Verwirrtheit und vorangegangene Stürze zu den Dispositionen, welche die Wahrscheinlichkeit von Fixierungsmaßnahmen erhöhen [6]. Die Angst vor der Entfernung lebenswichtiger arterieller oder venöser Zugänge durch den Patienten ist für Ärzt(innen) und Pfleger(innen) ein anderes wichtiges Motiv, bewegungseinschränkende Maßnahmen als notwendig zu erachten und zu veranlassen [36]. Studien belegen hingegen, dass es trotz mechanischer Fixierung zu Zwischenfällen kommen kann, bei denen sich die Patienten Zugänge entfernen, jedoch in den seltensten Fällen mit schweren bzw. lebensbedrohlichen Folgen [37]. Die fehlende Compliance bei mechanischen Fixierungen tritt insbesondere bei Patienten mit Demenzen auf, da diese die Notwendigkeit medizinischer Maßnahmen unter Umständen nicht mehr nachvollziehen können und sich aus einer für sie unangenehmen Lage befreien möchten [7].

Unter bestimmten Umständen können bewegungseinschränkende Maßnahmen als solche auch Verletzungen verursachen. Bettgitter vergrößern bspw. die Sturzhöhe und somit die Wahrscheinlichkeit für schwere Verletzungen [24]. Des Weiteren besteht vor allem bei mechanischen Fixierungen die Möglichkeit des Auftretens von Druckgeschwüren, Ödemen und Kontrakturen [10]. Die Gefahr von Inkontinenz, Verstopfung, Mangelernährung, Verringerung der Muskelkraft und des Gleichgewichts sowie eine verstärkte Abhängigkeit bei alltäglichen Verrichtungen, die durch beide Arten der Fixierung hervorgerufen werden können, erhöht sich [7]. Ursache hierfür kann die unsachgemäße Anbringung durch das Personal sein oder der Versuch von Patienten, sich die Fixierung selbst zu entfernen. Aber auch die andauernde oder wiederkehrende Immobilisation [25] der Patienten durch mechanische oder chemische Bewegungseinschränkungen kann die aufgeführten Folgen haben.

\section{Medizinethische Analyse}

Im Zusammenhang mit bewegungseinschränkenden Maßnahmen stellt sich die Frage, ob deren Durchführung ethisch vertretbar und zu rechtfertigen ist, und welche Bedeutung dies für die neue gesetzliche Regelung in der Schweiz hat. Es sollte hervorgehoben werden, dass die rechtliche Normierung einer bestimmten Handlung nicht gleichbedeutend mit deren ethischer Richtigkeit ist [52]. Demzufolge ist es wichtig, gesetzliche Regelungen zu hinterfragen und deren Auswirkungen für die betroffenen Personen zu analysieren. Die Angemessenheit des Einsatzes von bewegungseinschränkenden Maßnahmen spielt eine bedeutende Rolle. Es ist zu klären, ob es Fälle gibt, in denen Bewegungseinschränkungen angebracht sind, und falls ja, unter welchen Voraussetzungen. Auch wäre zu analysieren, ob solche Kasuistiken vom neuen Gesetz erfasst werden.

\section{Prinzipienethik}

Im Rahmen der Prinzipienethik, deren Grundlagen 1979 durch das Buch von Beauchamp und Childress „Principles of Biomedical Ethics“ gelegt wurden, können die folgenden Prinzipien zur Bearbeitung des Problems herangezogen werden: 1) Verpflichtung, keinen Scha- 
den zuzufügen (Nicht-Schadens-Prinzip), 2) Verpflichtung, Gutes zu tun (Benefizienz) [3]. Demnach soll dem Patienten kein Schaden zugefügt werden und dessen Gesundheit, Wohlergehen sowie seine Sicherheit gefördert und selbstschädigenden Verhaltensweisen entgegengewirkt werden [57]. Das Fürsorgeprinzip soll den Patienten schützen. Eine zu starke Ausweitung dieser Pflicht kann allerdings unter Umständen zu paternalistischen Verhaltensweisen führen, welche die Autonomie des Patienten einschränken. Dieser Prinzipienkonflikt ist insbesondere im Fall von bewegungseinschränkenden Maßnahmen problematisch. Aus Angst, der Patient könnte sich durch das Entfernen eines arteriellen oder venösen Zugangs oder durch unkontrollierte Bewegungen verletzen oder schädigen, werden vorsorglich Fixierungen angeordnet [5]. Derartige Maßnahmen führen unter Umständen jedoch auch zu Schädigungen des Patienten, was ursprünglich verhindert werden sollte. Die - vor allem bei mechanischen Fixierungen - entstehenden Verletzungen können nicht nur körperlicher Art sein, sondern auch psychische Folgen haben, etwa Vertrauensverlust gegenüber Pflegekräften und medizinischem Personal bewirken oder Panikattacken auslösen [19]. Hier verdeutlicht sich das eigentliche Dilemma, welches das medizinische Personal zu bewältigen hat: Das Wohl des Patienten soll durch eine Maßnahme gewährleistet werden, gleichzeitig kann genau diese Maßnahme Sicherheit und Gesundheit des Patienten auf vielfältige Weise gefährden [6]. Solche Folgen widersprechen eindeutig der Verpflichtung, Gutes zu tun. Eine medizinische Maßnahme mit derart zwiespältigen Auswirkungen in Bezug auf das Wohlbefinden des Patienten sollte daher nur in sehr engen Grenzen durchgeführt werden.

Als dritter Punkt muss der Respekt vor der Autonomie des Patienten genannt werden. ${ }^{1}$ Wiesemann stellt treffend fest, dass Autonomie als moralisches Recht zu einem Schlüsselbegriff moderner Gesellschaften avanciert ist [56]. Insbesondere im Rahmen der Entwicklung der modernen Medizinethik wurde das Konzept der Autonomie in den Vordergrund gestellt. Die Entscheidungsfreiheit des Patienten über medizinische Eingriffe jeglicher Art stellt die bedeutsamste und weitreichendste Neuerung der Selbstbestimmung dar. Menschliche Wesen sind keine Objekte, sondern Träger ihrer individuellen Entscheidungen [44] und Experten ihres eigenen Lebens [56]. Dies soll jedoch nicht heißen, dass sie Entscheidungen ohne Relation zu anderen Mitmenschen treffen müssen [2]. In Bezug auf die Vornahme von Fixierungsmaßnahmen kann insbesondere die Bedeutung der Autonomie als Abwehrrecht zum Tragen kommen [44]. Das Autonomieprinzip bekommt eine moralische Relevanz für das medizinische Personal, mit dem mögliche paternalistische Verhaltensweisen eingedämmt werden sollen [42]. Steinfath/Pindur betonen die Wichtigkeit der Einbeziehung des institutionellen und interpersonalen Kontextes, um die Fähigkeit von Patienten, autonom entscheiden zu können, zu stärken. Des Weiteren sollten die Persönlichkeit, der Charakter und die Fähigkeiten des Patienten eine besondere Bedeutung spielen, um dessen Wünsche und Verhaltensweisen besser nachvollziehen zu können, damit die Urteilsfähigkeit nicht vorschnell aberkannt wird [49]. Auch leiblichen Ausdrucksformen (Gesten/Bewegungen, die z. B. Wohlsein/Unwohlsein, Ablehnung/Zustimmung ausdrücken können,) sollte vor allem im pflegerischen Bereich mehr Beachtung geschenkt werden, da sie Befindlichkeiten und Willensbekundungen ausdrücken können, und sich die Selbstbestimmung ansonsten nur auf die Zustimmung zu helfenden Handlungen reduziert [30].

\footnotetext{
${ }^{1}$ Der vierte Punkt der Gleichbehandlung/Gleichberechtigung (Justice) spielt im Zusammenhang von bewegungseinschränkenden Maßnahmen nur eine untergeordnete Rolle und wird daher nicht in die Beurteilung einbezogen.
} 
Nun ist fraglich, wie sich das Autonomiekonzept mit der Durchführung von Fixierungen bei Patienten vereinbaren lässt. Handelt es sich um eine Willensäußerung, wenn sich der Patient arterielle oder venöse Zugänge oder andere medizinische Hilfsmittel entfernt? Muss diese von Ärzten und Pflegepersonal respektiert werden, obwohl Zweifel an einer selbstbestimmten Entscheidung bestehen, weil sich der Patient in einem kritischen Zustand befindet oder bereits die Urteilsunfähigkeit festgestellt wurde? Oder ist eine derartige Überlegung das Ergebnis eines ausgeuferten Autonomieverständnisses? Fest steht, dass eine Willensäußerung nicht pauschal ausgeschlossen werden sollte. Es ist wichtig, dass allen Patienten das höchstmögliche Maß an Autonomie zugestanden wird.

Die individuelle Beurteilung des Sachverhaltes aller äußeren Umstände und das Wohl des Patienten sollten für die abschließende Entscheidung für oder gegen eine bewegungseinschränkende Maßnahme ausschlaggebend sein. Schlussfolgernd kann gesagt werden, dass sowohl das Nicht-Schadens-Prinzip als auch das Prinzip, Gutes zu tun, und das Prinzip des Respekts der Patientenautonomie grundsätzlich gegen Fixierungsmaßnahmen sprechen.

\section{Pflegeethik/Fürsorgeethik}

In der Pflegeethik spielt das Fürsorgeprinzip eine zentrale Rolle [17]. Gastmans et al. bezeichnen die Idee der ,guten Pflege“ als das ultimative Ziel der pflegerischen Praxis. Pflege bedeute immer auch, die Fähigkeiten des Patienten zu fördern. Außerdem beschreiben die Autoren die Tugend der Fürsorge als einen inhärenten Bestandteil der moralischen Praxis in der Pflege [18]. Die Fürsorgeethik fokussiert sich auf Personen, welche in der Pflege in einer Beziehung zueinander stehen, und hebt somit das relationale Verhältnis zwischen Menschen hervor. Bei der Durchführung von bewegungseinschränkenden Maßnahmen spielt die Arzt ${ }^{2}$-Pfleger-Patient-Beziehung eine bedeutende Rolle und ist für die moralische Qualität ihres Handelns maßgeblich. Pflege ist eine Art, die andere Person anzuerkennen, und bezweckt hauptsächlich die Würde des Menschen [53].

Würde des Menschen

Die Würde des Menschen ist unantastbar, sie kann nicht verloren gehen, nicht einmal durch Krankheit, Behinderung oder den nahenden Tod. Dennoch handelt es sich um einen Begriff, dessen Gehalt und Anwendungsbereich strittig sind [15]. Speziell die „Würdelosigkeit eines Zustandes“ und die Verletzung der Menschenwürde werden als Argumente für oder gegen bestimmte medizinische Handlungsweisen angeführt. Ein häufig zitiertes Beispiel ist einerseits die Debatte um die Sterbehilfe: Die gemutmaßte Würdelosigkeit einer schwer kranken, sterbenden Person verlangt demnach ein bestimmtes Handeln [14]. Andererseits stellt sich die Frage, ob die Durchführung einer bestimmten Maßnahme - der Fixierung - eine Handlung darstellt, welche die Würde des Menschen verletzt. Im Rahmen einer bewegungseinschränkenden Maßnahme ist es der betroffenen Person meist nicht möglich, einfache alltägliche Handlungen, wie z. B. sich an der Nase zu kratzen, aufzustehen oder etwas zu trinken, selbstständig durchzuführen.

Ungewiss ist, ob diese Kriterien genügen, damit eine Verletzung der Menschenwürde vorliegt. Denn durch die Bewegungseinschränkung wird verhindert, dass der Patient sich

${ }^{2} \operatorname{Im}$ Folgenden gelten die verwendeten Begriffe und Bezeichnungen in männlicher Form analog für Frauen. 
selbst Schaden zufügt. Der möglicherweise entwürdigenden Maßnahme steht also eine vermeintlich gesundheitserhaltende oder gesundheitsverbessernde Handlung gegenüber. Rechtfertigt dies eine, wenn auch nur kurzzeitige, Verletzung der Menschenwürde? Eine bewegungseinschränkende Maßnahme kann nicht per se als Würdeverletzung gelten, aber es kann Konstellationen geben, in denen dies zutrifft. Deshalb sollten Bewegungseinschränkungen als Maßnahmen, welche die Würde des Menschen einschränken können, eingestuft werden und nur angewandt werden, wenn es keine andere Lösung gibt, d. h. ein Patient auf keine andere Weise von selbst- und fremdschädigenden Handlungen abgehalten werden kann, wie z. B. ein Patient im Delir.

\section{Informierte Einwilligung}

Wenn bewegungseinschränkende Maßnahmen in Betracht gezogen werden, muss der Patient so ausführlich wie möglich über deren Vorteile, aber auch deren mögliche Risiken informiert werden. Der betroffene Patient muss die Erläuterung verstehen und in die Maßnahme einwilligen. Daraus folgt, dass Bewegungseinschränkungen bei urteilsfähigen Personen immer erst nach Einwilligung erfolgen dürfen. Willigt der Patient nicht in eine Maßnahme ein, darf sie nicht durchgeführt werden. Für diese Art der Einwilligung im Zusammenhang mit Bewegungseinschränkungen gibt es keine gesetzliche Regelung.

Problematisch kann die Einschätzung der Urteilsfähigkeit sein. Bei urteilsunfähigen Patienten wird, laut Artikel 384 ZGB, die bei medizinischen Maßnahmen zur Vertretung berechtigte Person über die mechanische Bewegungseinschränkung informiert. Auch wenn die Einbeziehung von Vertretern in medizinische Entscheidungen gängige Praxis ist, kann sie ethische Probleme aufwerfen. Dies ist in besonderem Maße der Fall, da es sich um eine faktische Fremdbestimmung handelt und Missbrauchsrisiken nicht vollständig ausgeschlossen werden können [51]. Außerdem ist zu beachten, dass dem mutmaßlichen Patientenwillen durch den Stellvertreter nur mittelbar Ausdruck verliehen werden kann [50].

\section{Analyse der gesetzlichen Regelung}

Bewegungseinschränkende Maßnahmen sind eine Art der Freiheitsentziehung und bedürfen daher immer einer Rechtfertigung. Die Frage nach der Indikation für eine Maßnahme ist abzuklären, da der standardmäßige Gebrauch in der medizinischen Versorgung von Patienten kein Behandlungsziel sein kann, sondern immer eine Ausnahme darstellen sollte [27]. Gesetze können in diesem Fall eine Richtschnur sein und für Klarheit sorgen.

Die Bewegungsfreiheit wird in der Schweizer Bundesverfassung (BV) gemäß Artikel 10 Absatz 2 geschützt. Fraglich ist daher, aus welchem Grund, trotz einer vorhandenen bundesgesetzlichen Regelung, eine zusätzliche zivilgesetzliche Regelung eingeführt wurde. Das Ziel, welches mit dieser neuen gesetzlichen Regelung zu bewegungseinschränkenden Maßnahmen verfolgt wird, ist die Autonomie und die persönliche Freiheit von Patienten zu stärken [9]. Außerdem soll sie einen verstärkten Schutz urteilsunfähiger Personen in Heimen gewährleisten [41]. Es kann demgemäß geschlussfolgert werden, dass die ursprüngliche gesetzliche Regelung auf Bundesebene die nötigen Schutzanforderungen nicht gewährleisten konnte, welche für diesen sensiblen Bereich notwendig sind. 
Der neue Artikel 383 ZGB beinhaltet eine detaillierte Regelung der „Einschränkung der Bewegungsfreiheit":

1. Die Wohn- oder Pflegeeinrichtung darf die Bewegungsfreiheit der urteilsunfähigen Person nur einschränken, wenn weniger einschneidende Maßnahmen nicht ausreichen oder von vornherein ungenügend erscheinen und die Maßnahme dazu dient:

1. eine ernsthafte Gefahr für das Leben oder die körperliche Integrität der betroffenen Person oder Dritter abzuwenden; oder

2. eine schwerwiegende Störung des Gemeinschaftslebens zu beseitigen.

2. Vor der Einschränkung der Bewegungsfreiheit wird der betroffenen Person erklärt, was geschieht, warum die Maßnahme angeordnet wurde, wie lange diese voraussichtlich dauert und wer sich während dieser Zeit um sie kümmert. Vorbehalten bleiben Notfallsituationen.

3. Die Einschränkung der Bewegungsfreiheit wird so bald wie möglich wieder aufgehoben und auf jeden Fall regelmäßig auf ihre Berechtigung überprüft.

Der Artikel besteht aus drei Absätzen: Der erste Absatz bezieht sich auf die Voraussetzungen für die Einschränkung der Bewegungsfreiheit bei urteilsunfähigen Personen. Dies liegt darin begründet, dass urteilsfähige Personen selbst in die Maßnahme einwilligen können und daher keiner gesetzlichen Regelung bedürfen. Der Artikel bezieht sich explizit auf die Einschränkung der Bewegungsfreiheit in Wohn- oder Pflegeeinrichtungen. Hierbei ist anzumerken, dass demzufolge bewegungseinschränkende Maßnahmen in Krankenhäusern und im Rahmen der häuslichen Pflege nicht unter diese Regelung fallen. Zumindest lässt sich auf den ersten Blick nicht erkennen, wie der Begriff Wohn- oder Pflegeeinrichtung auszulegen ist und ob Krankenhäuser im Rahmen einer weiten Auslegung als Pflegeinrichtung eingestuft werden können. Das gleiche gilt für den Begriff Wohneinrichtung im Fall der häuslichen Pflege. Des Weiteren darf eine bewegungseinschränkende Maßnahme nur ergriffen werden, wenn die urteilsunfähige Person entweder ihr eigenes Leben, ihre körperliche Integrität oder Dritte ernsthaft gefährdet, oder wenn sie das Gemeinschaftsleben auf schwerwiegende Weise stört. Das heißt, dass die normativen Kriterien für eine Maßnahme dieser Art sehr eng sind.

Im zweiten Absatz des Artikels ist die Aufklärung des Patienten gesetzlich festgeschrieben, womit auch der Respekt vor urteilsunfähigen Personen deutlich gemacht wird. Demnach werden bewegungseinschränkende Maßnahmen nicht einfach angeordnet und durchgeführt, sondern der Patient wird trotz seiner Urteilsunfähigkeit über das Prozedere informiert. Im Rahmen einer vertrauensvollen Arzt-Patienten- bzw. Pfleger-PatientenBeziehung ist die Förderung der Kommunikation bei der Durchführung von bewegungseinschränkenden Maßnahmen ein wichtiger Bestandteil.

Im dritten Absatz wird die zeitliche Begrenzung für Bewegungseinschränkungen angesprochen. Die Aussagen bleiben dabei allerdings sehr vage und es gibt keine Zeitangaben, wann und wie oft eine Maßnahme überprüft werden muss. Insbesondere bleibt unklar, was so bald wie möglich/regelmäßig bedeutet. Dies kann für Pflegende ein praktisches Problem werden, da es im Gesetz keine Hinweise auf Maximalzeiträume gibt. Demnach scheint es momentan in der Verantwortung der Betreuenden zu liegen, wann und wie oft eine bewegungseinschränkende Maßnahme überprüft wird. 
In einem weiteren Artikel (384 ZGB) wird zum einen die Protokollierungspflicht (Name der anordnenden Person, Zweck, Art und Dauer der Maßnahme) für bewegungseinschränkende Maßnahmen, zum anderen die Informationspflicht gegenüber der bei medizinischen Maßnahmen zur Vertretung berechtigten Person beschrieben. Daraus lässt sich schließen, dass die alleinige Übermittlung einer Information kein Mitspracherecht beinhaltet. Um die Legitimität von Fixierungen mit Gurten etc. bei Urteilsunfähigkeit zu gewährleisten, dürfte nicht nur die Mitteilung über die Durchführung der Maßnahme ausreichen, sondern es müsste eine Einwilligung durch die zur Vertretung berechtigte Person erfolgen. Eine solche Regelung wäre Ausdruck für den Respekt vor der Autonomie und der Würde des Patienten [15]. Zwar liegt im Wirken eines Stellvertreters stets auch ein Moment der Fremdbestimmung, die Privatautonomie würde dadurch dennoch stärker gewahrt als durch die Fremdbestimmung durch Dritte (Ärzte, gerichtlich bestellte Betreuer) [32]. Ob die aktuelle gesetzliche Regelung den gängigen ethischen Standards in der medizinischen Praxis entspricht, ist deshalb in Frage zu stellen. Zwar ist die schriftliche Anrufung der Erwachsenenschutzbehörde gemäß Artikel 385 ZGB jederzeit möglich, dessen ungeachtet ist damit ein administrativer Aufwand verbunden, welchen nicht alle Menschen gleichermaßen zu bewältigen vermögen. Der Gesetzgeber sollte folglich auf die individuellen Lebenssituationen der Angehörigen Rücksicht nehmen. Im Zuge der Umsetzung der Gesetzesnovelle zu bewegungseinschränkenden Maßnahmen könnten Anlaufstellen eingerichtet werden, die Betroffenen konkrete Unterstützung anböten und bspw. telefonische Anfragen entgegennähmen.

Eine Besonderheit der aktuellen Gesetzgebung stellt die getrennte Regulierung von Bewegungseinschränkungen durch mechanische Hilfsmittel und durch sedierende Medikamente dar. Der Gesetzgeber hat sich dafür entschieden, die beiden Arten der Bewegungseinschränkung nicht gleichartig zu behandeln. Dies kann Vorteile und Nachteile haben. Auf der einen Seite wird deutlich, dass mechanische und chemische Bewegungseinschränkungen gleichermaßen einen Regelungsbedarf erfordern, auf der anderen Seite macht diese höhere Vorschriftendichte die praktische Arbeit im pflegerischen Alltag diffiziler, da unterschiedliche Regelungen beachtet werden müssen. Chemische Fixierungen fallen demnach nicht unter Artikel 383 ZGB. Bei der chemischen Bewegungseinschränkung findet Artikel 377 ff. ZGB Anwendung und die vertretungsberechtigte Person wird in die Entscheidung einbezogen [8]. Diese Zweiteilung vermittelt den Eindruck, dass für den Gesetzgeber die medikamentöse Bewegungseinschränkung einen grundlegend anderen Charakter aufweist als die Bewegungseinschränkung mit Hilfe von Fixierungsgurten, Gittern oder Netzen. Ob dies eventuell an möglichen Nebenwirkungen bei der Gabe von sedierenden Medikamenten liegt, bleibt aufgrund des Gesetzestextes unklar.

\section{Bewertung}

Bewegungseinschränkende Maßnahmen in der Medizin sollten ausschließlich oder mindestens immer im Interesse des betroffenen Patienten sein. Dabei kann es sich um Notfallmaßnahmen oder um präventive Maßnahmen handeln. Der Nutzen dieser medizinischen Maßnahmen ist indes nicht klar, da es sich um eine bisher nicht-validierte Therapie handelt [5]. Das persistierende Fehlen eines Nachweises der Effektivität in der klinischen Praxis verdeutlicht die Brisanz der Maßnahmen. Qualitativ hochwertige wissenschaftliche Studien zur Minimierung von Fixierungen wären notwendig, um zu untersuchen, ob die Verrin- 
gerung von Fixierungen nicht automatisch mit dem Risiko von Therapieunterbrechungen verbunden sein muss bzw. ob das Risiko von Verletzungen durch Fixierungen selbst viel höher ist [36].

Gesetzliche Regelungen können die weitere Entwicklung in diesem Bereich beeinflussen, wie Beispiele aus Schottland und Dänemark zeigen, wo die Fixierung älterer Menschen verboten ist [22]. In Österreich betreffen die Regelungen des „Heimaufenthaltsgesetzes“, welches 2005 in Kraft getreten ist, chemische und mechanische Bewegungseinschränkungen gleichermaßen [33]. Ein besonderes Merkmal der gesetzlichen Regelung in der Schweiz hingegen ist, wie oben bereits erwähnt, die Trennung von mechanischen und chemischen Fixierungen. Ethisch ist diese Trennung kaum zu rechtfertigen, da beide Arten der Bewegungseinschränkung die gleichen Konsequenzen für urteilsunfähige Patienten haben und daher auch gleich behandelt werden sollten. Eine einheitliche Lösung im Umgang mit bewegungseinschränkenden Maßnahmen hätte zudem zu einer Harmonisierung beigetragen und wäre auch deshalb vorzuziehen gewesen.

Wie medizinethische Analysen zeigen, lassen sich bewegungseinschränkende Maßnahmen nur sehr selten ethisch rechtfertigen. Oftmals widersprechen sie den gängigen medizin- und pflegeethischen Prinzipien. Die therapeutischen Vorteile wiegen die negativen Auswirkungen in den wenigsten Fällen auf. Folglich ist die moralische Vertretbarkeit der Anwendung einer Maßnahme an sich nicht gegeben. Die mechanischen oder chemischen Fixierungen von Patienten werden teilweise aus Gründen wie Zeit-, Personal- oder Ressourcenmangel durchgeführt, können diese aber keinesfalls rechtfertigen [8]. Eine einfache Lösung für viele Probleme scheint gängige Attitüde gegenüber Fixierungsmaßnahmen im Rahmen der Gesundheitsversorgung zu sein [12].

Ferner wäre es sinnvoll gewesen, mit Hilfe dieser gesetzlichen Regelung einen einheitlichen Standard innerhalb und auch zwischen verschiedenen Einrichtungen einzuführen. Patienten, die von Fixierungen betroffen sind, sollten nicht aufgrund von subjektiven oder vielleicht sogar willkürlichen Einschätzungen des medizinischen und pflegerischen Personals ungleich behandelt werden. Eindeutige Zeitangaben im Zusammenhang mit der Überprüfungspflicht der Fixierungsmaßnahmen gemäß Artikel 383 ZGB erster Halbsatz hätten Unsicherheiten in diesem Bereich vermieden. Die Aufhebungspflicht (regelmäßige Überprüfung der Maßnahme) laut Artikel 383 ZGB zweiter Halbsatz lässt sich weiterhin nur mit Blick auf den konkreten Einzelfall verantworten. Ohne strikte Vorgaben zur Regelmäßigkeit der Überprüfung und der damit verbundenen möglichen Aufhebung der Fixierung wird das Wohl der Patienten in die Hände der Verantwortlichen gelegt. Dies könnte die Wahrscheinlichkeit von Missbrauch in Einrichtungen mit personeller Unterbesetzung erhöhen. Der Gesetzgeber überlässt damit den Gerichten die genaue Definition der fraglichen Begrifflichkeiten und zeitlichen Begrenzungen, sollte es diesbezüglich zu einem Rechtsstreit kommen, und entzieht sich in diesem Fall in gewissem Maße seiner Verantwortung. Überdies ist ungewiss, ob die Fähigkeit der involvierten Personen, diesbezüglich eine adäquate Entscheidung treffen zu können, überhaupt vorausgesetzt werden kann. Es ist zu vermuten, dass der Gesetzgeber davon ausgeht, dass Personen, die bewegungseinschränkende Maßnahmen durchführen, dazu in der Lage sind, die zeitlichen Überprüfungsabstände richtig einzuschätzen. In einem hochsensiblen medizinischen und pflegerischen Bereich kann eine Annahme dieser Art jedoch zu ungewollten Zuständen führen.

Eine bewegungseinschränkende Maßnahme kann nur dann angemessen sein, wenn eine Güterabwägung zeigt, dass es keine andere Möglichkeit als die Bewegungseinschränkung 
gibt, um den Patienten vor sich selbst oder anderen Personen zu schützen, oder wenn der Patient in die Maßnahme einwilligt, da er/sie sich beispielsweise mit einem Bettgitter in der Nacht sicherer fühlt [26]. Generell dürfen ohne die Prüfung der Verhältnismäßigkeit keine Bewegungseinschränkungen durchgeführt werden. Konkret muss begutachtet werden, ob die Maßnahme notwendig und proportional ist und nicht durch eine andere Maßnahme ersetzt werden kann. In Anlehnung an eine Entscheidung des deutschen Bundesverfassungsgerichtes zu Zwangsbehandlung im Maßregelvollzug sollten bewegungseinschränkende Maßnahmen gegen den Willen des Patienten demnach „,nur dann eingesetzt werden, wenn sie im Hinblick auf das Behandlungsziel, das ihren Einsatz rechtfertigt, Erfolg versprechen und für den Betroffenen nicht mit Belastungen verbunden sind, die außer Verhältnis zu dem erwarteten Nutzen stehen. ${ }^{\text {"3 }}$ Ein besonders wichtiger Punkt ist hierbei die Kommunikation mit dem Patienten [11], um dessen Verständnis für die Maßnahme und somit seine Compliance zu erhöhen. Auch wenn Freiheitsbeschränkungen kaum aus dem Pflegealltag wegzudenken sind [24], sollte deren Anwendung grundsätzlich nur in Ausnahmefällen in Betracht gezogen werden (ultima ratio). Sie sollten nicht als Routinemaßnahme eingesetzt werden und die am wenigsten eingreifende Maßnahme muss Vorrang haben. Diese Aspekte werden in den neuen Artikeln des ZGB berücksichtigt, finden jedoch nach der Wortlautauslegung nur Anwendung für Wohn- oder Pflegeeinrichtungen. Folglich werden, zumindest nach dem Wortlaut, Krankenhäuser oder psychiatrische Einrichtungen ausgeschlossen.

Alternativen zur Fixierung können bspw. die Verwendung von Hüftprotektoren, AntiRutsch-Matten usw. darstellen. Des Weiteren rücken viele Studien Bildungsmaßnahmen wie Workshops oder Seminare, Fallbeispiele und Diskussionen zur Minimierung von Fixierungen in den Vordergrund. Leider sind deren Herangehensweisen und die Ergebnisse sehr unterschiedlich, ein eindeutiger und langfristiger Effekt lässt sich daher nicht attestieren [39].

Das Joanna Briggs Institute hat bereits 2002 eine Auflistung von Fixierungsalternativen erarbeitet. Es werden unter anderem: a) Veränderungen in der Umgebung mit beispielsweise verbesserten Lichtverhältnissen, rutschfesten Bodenbelägen, barrierefreie Raumarchitektur; b) Sicherheit im Bett mit konkaven Matratzen, Einsatz von Seitenschläferkissen, Matratzen vor dem Bett als Abfederung bei Stürzen; c) Aktivitäten und Programme wie Physio- und Ergotherapie, Fitnessübungen, Beschäftigungen/Betreuungsmöglichkeiten für Nachtwandler; d) Änderungen in der Pflege, wie z. B. Erhöhung des Personalspiegels, gefährdete Patienten in der Nähe der Pflegestation, schnellstmögliches Entfernen von Kathetern, Drainagen etc., vorgeschlagen [31]. Auch das Abdecken von Zugängen durch Mullbinden wäre eine Möglichkeit, das Entfernen derselben zu verhindern [7], bzw. würde dem Pflegepersonal genügend Zeit lassen einzugreifen, falls sich der Patient unwohl fühlen sollte.

\section{Schlussüberlegung}

Die Güterabwägung zeigt, dass bewegungseinschränkende Maßnahmen nur kurzfristig und nur unter ganz bestimmten Voraussetzungen durchgeführt werden sollten. Die neue gesetzliche Regelung in der Schweiz kann als ein erster Schritt in die richtige Richtung betrachtet werden. Sie gibt eine Orientierung für das medizinische Personal, aber auch für Angehörige von betroffenen Patienten. Leider bestehen Regelungslücken, welche Unsicherheiten oder

${ }^{3}$ BVerfGE 128, 282 
Unklarheiten in der Praxis hervorrufen können und so einen schweizweiten, einheitlichen Gebrauch erschweren. Eine Möglichkeit zur Vorbeugung wäre z. B. eine Liste mit spezifischen Indikationen, wann Fixierungsmaßnahmen angewendet werden dürften [48]. Bewegungseinschränkende Maßnahmen sollten generell nur so wenig wie möglich angewendet werden, um Grundrechtsverletzungen zu vermeiden. Außerdem sollte das medizinische Personal zu dieser Thematik geschult werden, um die Aufmerksamkeit für das Problem und die Pflegequalität zu erhöhen [55]. In Einrichtungen, in denen genügend Personal zur Verfügung steht und in denen es eine flexible Organisationsstruktur gibt, sind Fixierungen in den seltensten Fällen nötig, wie das Beispiel des Alters- und Pflegeheims in Stana, Kanton Wallis, belegt [43]. Ein Mittel zur Vorbeugung gegen bewegungseinschränkende Maßnahmen, und ergo ein Garant für den respektvollen Umgang mit pflegebedürftigen Patienten, wäre ausreichend Zeit.

Interessenkonflikt W. Bretschneider gibt an, dass kein Interessenkonflikt besteht.

Dieser Beitrag beinhaltet keine Studien an Menschen oder Tieren.

\section{Literatur}

1. Alexander FG, Selesnick ST (1969) Geschichte der Psychiatrie. Ein kritischer Abriss der psychiatrischen Theorie und Praxis von der Frühgeschichte bis zur Gegenwart. Diana, Konstanz

2. Anderson J (2013) Relationale Autonomie 2.0. In: Wiesemann C, Simon A (Hrsg) Patientenautonomie. Theoretische Grundlagen - Praktische Anwendungen. Mentis, Münster, S 61-75

3. Beauchamp T (2005) Prinzipien und andere aufkommende Paradigmen in der Bioethik. In: Rauprich O, Steger F (Hrsg) Prinzipienethik in der Biomedizin. Campus, Frankfurt, S 48-73

4. Bourbonniere M, Strumpf NE, Evans LK, Maislin G (2003) Organizational characteristics and restraint use for hospitalized nursing home residents. J Am Geriatr Soc 51:1079-1084

5. Bredthauer D (2006) Können Fixierungen bei dementen Altenheimbewohnern vermieden werden. Betreuungsmanag 4:185-191

6. Castle NG, Engberg J (2009) The health consequences of using physical restraints in nursing homes. Med Care 47:1164-1173

7. Cotter VT (2004) Restraint free care in older adults with dementia. Keio J Med 54:80-84

8. CURAVIVA (2012) Erwachsenenschutzrecht. Bewegungseinschränkende Massnahmen. CURAVIVA. http://upload.sitesystem.ch/131D5358A8/4BFEA0B204/230155FE95.pdf. Zugegriffen: 29. Jan. 2013

9. CURAVIVA (2012) Neues Erwachsenenschutzrecht. Basisinformationen, Arbeitshilfen und Musterdokumente für Alters- und Pflegeinstitutionen. CURAVIVA Schweiz, Fachbereich Alter. http://upload. sitesystem.ch/E262A04C9D/4BFEA0B204/6045A1F907.pdf. Zugegriffen: 20. Jan. 2013

10. Demir A (2007) Nurses' use of physical restraints in four Turkish hospitals. J Nurs Scholarsh 39:38-45

11. Duttge G (2013) Patientenautonomie und Einwilligungsfähigkeit. In: Wiesemann C, Simon A (Hrsg) Patientenautonomie. Theoretische Grundlagen - Praktische Anwendung. Mentis Verlag, Münster, S 77-90

12. Evans D, FitzGerald M (2002) Reasons for physically restraining patients and residents: a systematic review and content analysis. Int J Nurs Stud 39:735-743

13. Evans D, Wood J, Lambert L (2003) Patient injury and physical restraint devices: a systematic review. J Adv Nurs 41:274-282

14. Feldmann K (2013) Sterben, Sterbehilfe, Töten, Suizid. Bausteine für eine kritische Thanatologie und für eine Kultivierungstheorie, Version 259. http://www.feldmann-k.de/tl_files/kfeldmann/pdf/thantosoziologie/feldmann_sterben_sterbehilfe_toeten_suizid.pdf. Zugegriffen: 30. Jan. 2013

15. Fischer J (2007) Menschenwürde, Rationalität und Gefühl. In: Dalferth I, Hunziker A (Hrsg) Mitleid. Konkretionen eines strittigen Konzepts. Religion in Philosophy and Theology, Bd. 28. Mohr Siebeck Verlag, Tübingen, S 49-66

16. Flaherty JH (2004) Zero tolerance for physical restraints: difficult but not impossible. J Gerontol 59A:919-920 
17. Gastmans C (2006) The care perspective in healthcare ethics. In: Davis A, Tschudin V, de Reave L (Hrsg) Essentials of teaching and learning in nursing ethics. Perspectives and methods. Churchill Livingstone, Edinburgh, S 135-148

18. Gastmans C, Diercks de Casterle B, Schotsmans P (1998) Nursing considered as moral practice: a philosophical-ethical interpretation of nursing. Kennedy Inst Ethics J 8:43-69

19. Gastmans C, Milisen K (2006) Use of physical restraint in nursing homes: clinical-ethical considerations. J Med Ethics 32:148-152

20. Grosskopf V, Klein H (2002) Krankenpflege und Recht. 2. Aufl. Spitta, Balingen

21. Guerra V (2008) Fesselnde Sorge. Pflegebedürftige Menschen festzubinden ist ein Tabu - und gleichzeitig gängige Praxis. Das Projekt ReduFix bietet Aufklärung und Alternativen. Forum Sozialstation 151:44-46

22. Hamers FJ (2005) Why do we use physical restraints in the elderly? Z Gerontol Geriatr 38:19-25

23. Hamers JP, Meyer G, Köpke S, Lindenmann R, Groven R, Huizing AR (2009) Attitudes of Dutch, German and Swiss nursing staff towards physical restraint use in nursing home residents, a cross-sectional study. Int J Nurs Stud 46:248-255

24. Hansen R (2008) Auch Biografie in Entscheidungsfindung einbeziehen. Curaviva 6:8-11

25. Haut A, Kolbe N, Strupeit S, Mayer H, Meyer G (2010) Attitudes of relatives of nursing home residents toward physical restraints. J Nurs Scholarsh 42:448-456

26. Heinze C, Dassen T, Grittner U (2012) Use of physical restraints in nursing homes and hospitals and related factors: a cross sectional study. J Clin Nurs 21:1033-1040

27. Henke F (2006) Fixierungen in der Pflege. http://www.carelounge.de/pflegeberufe/autoren/artikel_ ansehen.php?id=63. Zugegriffen: 20. Jan. 2013

28. Hoffmann B, Klie T (2004) Freiheitsentziehende Massnahmen. Unterbringung und unterbringungsähnliche Massnahmen in Betreuungsrecht und Praxis. 1. Aufl. C.F. Müller, Heidelberg

29. Hofmann H, Hahn S (2013) Characteristics of nursing home residents and physical restraint: a systematic literature review. J Clin Nurs. doi:10.1111/jocn.12384

30. Hofmann I (2013) Leibliche Ausdrucksformen als Zeichen der Selbstbestimmung. In: Wiesemann C, Simon A (Hrsg) Patientenautonomie. Theoretische Grundlagen - Praktische Anwendungen. Mentis Verlag, Münster, S 355-363

31. Joanna Briggs Institute (2002) Physical restraint - part 2: minimisation in acute and residential care facilities. Best Pract 6:1-6

32. Kopetzki C (2004) Einleitung und Abbruch der künstlichen Ernährung beim einwilligungsunfähigen Patienten. Ethik Med 16:275-287

33. Mann E, Köpke S, Haastert B, Pitkälä K, Meyer G (2009) Psychotropic medication use among nursing home residents in Austria: a cross-sectional study. BMC Geriatr 9:18

34. Meyer G, Köpke S, Haastert B, Mühlhauser I (2009) Restraint use among nursing home residents: cross-sectional study and prospective cohort study. J Clin Nurs 18:981-990

35. Meyer G, Möhler R, Köpke S (2009) Interventions for preventing and reducing the use of physical restraints in long-term geriatric care. Cochrane Database Syst Rev 1:1-6

36. Minnick AF, Mion LC, Leipzig R, Lamb K, Palmer RM (1998) Prevalence and patterns of physical restraint use in the acute care setting. J Nurs Adm 28:19-24

37. Mion LC (2008) Physical restraint in critical care settings: will they go away? Geriatr Nurs 29:421-423

38. Möhler R, Richter T, Köpke S, Meyer G (2011) Interventions for preventing and reducing the use of physical restraints in long-term geriatric care. Cochrane Database Syst Rev 2:1-26

39. Möhler R, Richter T, Köpke S, Meyer G (2012) Interventions for preventing and reducing the use of physical restraints in long-term geriatric care - a Cochrane review. J Clin Nurs 21:3070-3081

40. Moore K, Haralambous B (2007) Barriers to reducing the use of restraints in residential elder care facilities. J Adv Nurs 58:532-540

41. Nicole A-M (2009) Auswirkungen des neuen Erwachsenenschutzrechts auf Heime und ihre Bewohnenden. „Die Anforderungen entsprechen ethisch-fachlichen Grundsätzen“. Curaviva 5:4-7

42. Rehbock T (2000) Braucht die Pflege eine eigene Ethik? Pflege 13:280-289

43. Schmid C (2013) Grosse Veränderungen in Walliser Alters- und Pflegeheim. Ohne bewegungseinschränkende Massnahmen geht es auch. Curaviva 1:35-37

44. Schöne-Seifert B (2007) Grundlagen der Medizinethik. Kröner, Stuttgart

45. Schumacher C (1997) Freiheitsentziehende Maßnahmen mit mechanischen Mitteln bei der Betreuung gebrechlicher Menschen. Recht und Praxis der Fixierung. 1. Aufl. Bundesanzeiger, Köln

46. Schweizerische Akademie der Medizinischen Wissenschaften (2005) Zwangsmassnahmen in der Medizin. http://www.samw.ch/de/Ethik/Richtlinien/Aktuell-gueltige-Richtlinien.html. Zugegriffen: 20. Jan. 2013 
47. Sloane PD, Mathew LJ, Scarborough M, Desai JR, Koch GG, Tangen C (1991) Physical and pharmacologic restraint of nursing home patients with dementia: impact of specialized units. JAMA 265:1278-1282

48. Steinert T, Lepping P, Bernhardsgrütter R, Conca A, Hatling T, Janssen W, Keski-Valkama A, Mayoral F, Whittington R (2010) Incidence of seclusion and restraint in psychiatric hospitals: a literature review and survey of international trends. Soc Psychiatry Psychiatr Epidemiol 45:889-897

49. Steinfath H, Pindur A-M (2013) Patientenautonomie im Spannungsfeld philosophischer Konzeptionen von Autonomie. In: Wiesemann C, Simon A (Hrsg) Patientenautonomie. Theoretische Grundlagen Praktische Anwendungen. Mentis Verlag, Münster, S 27-41

50. Strätling M, Scharf V, Wulf H, Eisenbart B, Simon A (2000) Stellvertreterentscheidungen in Gesundheitsfragen und Vorausverfügungen von Patienten. Eine praxisorientierte Übersicht zu rechtlichen und ethischen Problemen bei der Behandlung nicht einwilligungsfähiger Personen. Anaesthesist 49:657-674

51. Strätling M, Scharf VE, Wedel C, Oehmichen F, Eisenbart B (2001) Möglichkeiten zur Verminderung rechtlicher und ethischer Probleme bei der Behandlung nicht einwilligungsfähiger oder von Entscheidungsunfähigkeit bedrohter Patienten. Vorschläge für eine konzentrierte Gesetzgebungsinitiative zur Reformierung des deutschen Betreuungsrechts bezüglich antizipierender Behandlungsanweisungen und Stellvertreterentscheidungen in Gesundheitsfragen. Medizinr 19:385-395

52. Sulmasy D, Sugarman J (2001) The many methods of medical ethics (or, thirteen ways of looking at a blackbird). In: Sulmasy D, Sugarman J (Hrsg) Methods in medical ethics. Georgetown University Press, Washington, D.C., S 3-19

53. Vanlaere L, Gastmans C (2007) A normative approach to care ethics: the contribution of the louvain tradition of personalism. In: Gastmans C, Diericksx K, Nys H, Schotsmans P (Hrsg) New pathways for European bioethics. Intersentia, Antwerpen Oxford, S 99-118

54. Wenger S (2008) Die Geschichte der Zwangsmassnahmen. „Es war ein geschlossenes System ohne Kontrolle“. Curaviva 6:26-29

55. Wenger S (2009) Wie aus dem alten Vormundschaftsrecht der neue Erwachsenenschutz wurde. Mehr Persönlichkeitsschutz, mehr Selbstbestimmung. Curaviva 5:26-29

56. Wiesemann C (2013) Die Autonomie des Patienten in der modernen Medizin. In: Wiesemann C, Simon A (Hrsg) Patientenautonomie. Theoretische Grundlagen - Praktische Anwendungen. Mentis Verlag, Münster, S 13-26

57. Wiesemann C, Biller-Andorno N (2005) Medizinethik und Patient-Arzt-Beziehung. In: Wiesemann C, Biller-Andorno N (Hrsg) Medizinethik. Für die neue AO. Thieme, Stuttgart, S 11-25 